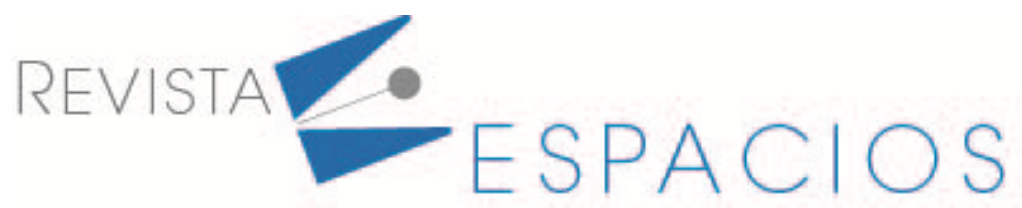

\title{
Evaluación de la cultura del diseño industrial en empresas familiares
}

\section{Evaluation of the culture of industrial design in family businesses}

\author{
GUANOLUISA, Robert. ${ }^{1}$ \\ CHIMBO, Christian. ${ }^{2}$ \\ ORTIZ, Luz. ${ }^{3}$ \\ SUÁREZ, Franyelit. ${ }^{4}$
}

\section{Resumen}

Se presenta una revisión de la cultura de diseño industrial en las empresas familiares de la ciudad de Quito, evaluando sus características y sus conocimientos en torno a la cultura de diseño industrial. Se utilizan modelos de análisis de diseño provenientes de países europeos adaptados a la realidad ecuatoriana. Fue posible evidenciar la importancia de la cultura del diseño industrial en la gestión y el mejoramiento de los procesos enfocados en el uso de nuevas tecnologías, innovación y valor agregado. Palabras clave: cultura del diseño, empresas familiares, valor agregado, innovación, gestión empresarial.

\begin{abstract}
A review of the industrial design culture in family businesses in the city of Quito is presented, evaluating their characteristics and their knowledge of industrial design culture. Design analysis models from European countries adapted to the Ecuadorian reality are used. It was possible to demonstrate the importance of industrial design culture in the management and improvement of processes focused on the use of new technologies, innovation and added value.

Key words: design culture, family businesses, added value, innovation, business management.
\end{abstract}

\section{Introducción}

El diseño industrial ha venido trascendiendo la historia para integrarse en los diferentes escenarios de las profesiones. Lo relativo a la industria está asociado a todo aquello que se produce, que empezó en el siglo XVIII con la revolución industrial. El diseño está asociado a los aspectos inherentes de los productos, su forma, su aplicación, su uso, su relación con el entorno, su participación con la sociedad, su relación con el ambiente y con los factores económicos, entre tantos aspectos propios de un producto industrializado.

\footnotetext{
${ }^{1}$ Investigador. Facultad de Ingeniería y Ciencias Aplicadas. Universidad Central del Ecuador. rjguanoluisa@uce.edu.ec

2 Investigador. Doctorado en Diseño, Fabricación y Gestión de Proyectos Industriales. Universidad Politécnica de Valencia. chchina@doctor.upv.es

${ }^{3}$ Docente-Investigadora. Facultad de Ciencias Psicológicas. Universidad Central del Ecuador. leortize@uce.edu.ec

${ }^{4}$ Docente-Investigadora. Facultad de Ingeniería y Ciencias Aplicadas. Universidad de las Américas. Franyelit.suarez@udla.edu.ec
} 
La cultura del diseño está intrínsecamente ligada a la realidad empresarial, ya que el diseño está al frente de todo lo que hace la organización, desde la estrategia hasta la ejecución. También significa que los líderes e involucrados, y el personal entienden el valor del diseño, utilizándolo para impulsar al usuario hacia una experiencia perfecta (InVision, 2018). El diseño es un instrumento del que ya disponen los ejecutivos, pero ha sido subutilizado (Simón, 2011). La cultura del diseño como filosofía empresarial es generadora de innovación para validar su carácter de diseño y no replicación, incluyendo un análisis sistémico y contextual del usuario y la forma de uso que converge en una generación de valores propios.

Es así como el estudio del diseño industrial responde a la migración de los clientes y usuarios de un producto o servicio funcional a uno con valor, lo cual es una respuesta a la extensa oferta de productos y servicios a la que los usuarios tienen acceso de manera presencial y digital (Romero, 2017). En la actualidad el consumidor no compra bienes, sino funciones orientadas a solucionar necesidades o deseos de los usuarios y clientes. La compra no responde únicamente a color o forma, a un valor estético, sino a un conjunto de características que satisfacen los requerimientos de los compradores tales como: formas atractivas, calidad óptima, precio adecuado y la identificación del producto o servicio a un concepto del fabricante. Es decir, responden a buenas prácticas de diseño (Iváñez, 2000).

La incorporación del diseño es un cambio cultural para las organizaciones y muchas veces no se produce de forma natural. Es allí donde los diseñadores son responsables de trabajar para generar una cultura de diseño que ayude a valorar el rol del diseñador, su importancia e impacto sobre generación de valor e innovación (Bustamante, 2019).

En este trabajo se presenta una revisión y evaluación bibliográfica de la cultura del diseño, definida como una estrategia para la generación de valor e innovación en las empresas familiares del área metropolitana de la ciudad de Quito, en Ecuador. El impacto económico de las empresas familiares para el mundo es relevante, así como las consecuencias en la competitividad y en el desarrollo de los sectores económicos (Ruíz, 2020). En Quito, las empresas familiares representan el 87,51\% del tejido empresarial (Camino \& Bermúdez, 2018).

Para esta investigación se consideraron los resultados expuestos por la Organización Mundial de la Propiedad Intelectual (Organización Mundial de la Propiedad Intelectual, 2015), que establecen rutas claras para medir la cultura de diseño en empresas bajo su nivel de innovación y generación de valor.

Este estudio está compuesto por cuatro secciones, en la primera se ha esbozado la naturaleza general y básica de la investigación desarrollada; luego se expone una sección referida a un análisis conceptual y bibliográfico de la cultura del diseño, innovación, generación de valor y análisis de la situación actual. Inmediatamente se encuentra la metodología llevada a cabo para este proceso. Para finalizar se exponen los resultados y las conclusiones.

\section{Planteamiento del problema}

Cuando nace la revolución industrial en el año 1760 (Gay \& Samar, 2007) se abren nuevos horizontes para la formulación de ideas de producción. Los productos que eran realizados de forma manual o artesanal pasan a elaborarse de forma mecánica e industrial, con ayuda de equipos y maquinarias que facilitan la construcción y agilizan los procesos. Es entonces cuando se separan las dos actividades fundamentales de la elaboración de un producto: la concepción y la construcción. Estos nuevos paradigmas llegarían a ser los elementos que trazarían el camino de las industrias modernas.

El mercado es cada vez más competitivo, por eso se hace más importante innovar. La innovación se ha convertido en el motor que impulsa la transformación y el crecimiento de las compañías (DESARROLLO MIC, 2017). En América Latina la percepción de la innovación como tema relevante para el desarrollo alcanza solo un $23 \%$ de 
aceptación como media y en Ecuador se obtiene $22 \%$ de aceptación de la premisa (Cañate \& Basco, 2018) (González, 2013).

El fracaso continuo en el proceso de innovación implica que se están produciendo pocos progresos en las prácticas de gestión de la innovación (Ortt \& Smits, 2006) (Balmaseda \& Zamanillo, 2008). La innovación empresarial puede ser de tipo organizacional, productivo o tecnológico, con el fin de ser más eficiente y conseguir una mejor posición el mercado o crear un mercado en el cual no haya muchos competidores (Fontalvo, Luckert, Martínez, \& Olivella, 2013).

En Ecuador se presentan escenarios interesantes que son debatibles. Algunos autores (Soliz, Mena, \& Núñez, 2017) aseguran que resulta necesaria la innovación, motivar a las organizaciones a una cultura de compartir, donde el conocimiento sea el eje central que asegure los beneficios a corto y largo plazo, pues este resulta un activo valioso para las empresas. De tal manera que las empresas familiares en el Ecuador deben fortalecer las iniciativas en torno al capital intelectual, que enriquezcan el crecimiento profesional y que se fundamenten en las bases de la investigación que harán posible una mejora de los productos y su participación en los mercados.

Es necesario conocer el ecosistema industrial para tomar acciones en torno al mejoramiento de los productos que harán posible su participación en los estándares internacionales, que se generen los ambientes necesarios para fomentar una cultura del diseño industrial, que promuevan nuevas formas de comercialización, de incorporación de los productos, que se construyan espacios para la innovación y la integración de ideas y desarrollos sostenibles.

Otros autores (Segundo, 2019) proponen un listado delimitado en el país para la mejora de los niveles de innovación empresarial como el acceso al crédito bancario a las micro, pequeñas y medianas empresas, políticas de innovación, acceso al comercio internacional, uso de las tecnologías de la información y mejorar la toma de decisiones empresariales. Se puede considerar que dentro de las tres últimas existe un nexo entre la generación de nuevos productos y el esparcimiento a nuevos mercados a través de una incorporación de sistemas tecnológicos y de decisión dentro de la empresa.

Basados en esta lista de acciones de mejora de proceso de producción, el enfoque de políticas de innovación en Ecuador según la encuesta Nacional de Actividades de Ciencia, Tecnología e Innovación (ACTI), Senescyt \& INEC: 1.206 (Instituto Nacional de Estadística y Censos, 2016), las empresas ecuatorianas destinaron recursos a la realización de alguna de las actividades conducentes a la innovación, lo que equivale a un 37\% de las compañías. Pero mayoritariamente, las innovaciones introducidas son incrementales, no rompen paradigmas, no le dan al producto o servicio final un valor agregado que permita su diferenciación en el mercado

Puede suponerse que los niveles de innovación en Ecuador no son los adecuados para enfrentar un desarrollo productivo y de expansión, lo que hace que sean deficientes como generadores de ventaja competitiva. Ecuador ocupa el número 99 de 126 países con un decrecimiento del 7.9\% en relación con el año 2017, observándose que países de características similares como Colombia y Perú ocupan los puestos 67 y 71 respectivamente (Organización Mundial de la Propiedad Intelectual, 2019).

La noción de innovación está implícita en la idea de diseño, éste implica necesariamente algún grado de novedad. Si el diseño no aportara ningún cambio a lo preexistente no es diseño, sino un proceso de replicación (Chaves, 2016). El diseño ha acelerado el ciclo de innovación empresarial, basando su importancia en la creación de nuevos productos o servicios que utilizan soluciones creativas para hacerse realidad (Barcelona Escola Superior de Disseny, 2016).

La OMPI (Organización Mundial de la Propiedad Intelectual, 2015) como promotor de innovación, ha motivado a la valoración del potencial del diseño en los países, con la intención de incrementar las innovaciones a través 
de la cultura del diseño. Esta iniciativa involucra empresas de Argentina y Marruecos en su mayoría, logrando importantes resultados en los diseños industriales, patentes y marcas que se convierten en agentes activos en la economía de los países (Jewell, 2016).

Basado en este contexto, el valor y la innovación presentan un gran aporte al crecimiento de productividad y competitividad de las empresas frente al mercado y competencia. Por la composición del tejido empresarial del Ecuador, según el primer informe sobre el estudio de la Empresa Familiar en Ecuador, presentado en la Universidad de Especialidades Espíritu Santo (UEES) el 90,5\% del tejido empresarial está conformado por Empresas Familiares (El Telégrafo, 2017). Las empresas familiares en muchas ocasiones se resisten al cambio y prefieren mantener lo que hasta ahora ha funcionado bien, o a conservar el sueño de su fundador y resistirse a realizar cambios en los procesos, productos o tecnologías que pueden llevar a la empresa a mejores niveles de competitividad (Vélez, 2008).

Este contexto motiva a la evaluación del potencial del diseño, como generador de valor e innovación en empresas familiares debido a su gran presencia dentro de las fibras empresariales de Ecuador. Se toma en cuenta en este trabajo el análisis de las empresas de la ciudad de Quito que han considerado la incorporación del diseño en su gestión empresarial y de qué manera esto afecta a la producción empresarial.

\section{Metodología}

La investigación presentada es de carácter descriptiva-transversal, ya que describe algunas características fundamentales de conjuntos homogéneos (Martínez, 2020). A nivel internacional se han realizado estudios que pueden revelar la significancia de la investigación. Sin embargo, los países latinoamericanos que presentan estudios similares cuentan con regulaciones y brechas en el ámbito del diseño a nivel de legislación fiscal, la incorporación del diseño en la mente de los habitantes y la historia de la inclusión del diseño como área del conocimiento.

La investigación transversal recoge la lista de empresas de la Superintendencia de Compañías del área metropolitana de Quito. Se fundamentaron y evaluaron estas teorías con una encuesta aplicada a líderes empresariales, accionistas, gerentes y encargados de empresas familiares. Para determinar la muestra del estudio se utilizó el directorio de empresas con corte en diciembre de 2019 de la Superintendencia de Compañías, Seguros y Valores del Ecuador. A través de una muestra probabilística simple y bajo los criterios de que las empresas se encuentren activas y tengan su sede principal dentro de los límites del área metropolitana de Quito. El componente descriptivo permite determinar la cantidad de tiempo y dinero que las empresas familiares del área metropolitana de Quito invierten en diseño, en qué medida es utilizado el diseño dentro de las empresas, el lugar que ocupa el diseño dentro de la toma de decisiones y la preferencia hacia áreas de diseño. Dicho resultado será correlacionado con los factores de innovación y generación de valor para aproximar su relación. Se evaluará también si las empresas familiares utilizan la cultura de diseño como estrategia para desarrollarse.

Además, la investigación es cualitativa ya que proporciona un esbozo sobre las empresas familiares y sus características actuales en torno a la cultura del diseño, incluyendo los aspectos de innovación y generación de valor (Fernández, 2017). Es posible también agregar que la investigación es de carácter conclusiva, debido a que no presenta una exploración del comportamiento de las empresas familiares hacia el diseño dentro de su entorno de relación y finalmente es posible afirmar que la investigación es de carácter transversal porque se realiza un corte de las empresas activas dentro del periodo 2019. 


\subsection{Muestreo}

La muestra es aleatoria simple y se obtuvo a partir de la ecuación (1)

$$
n=\frac{N \times Z_{a}^{2} \times p \times q}{d^{2} \times(N-1)+Z_{a}^{2} \times p \times q}
$$

Donde,

$\mathrm{N}=$ tamaño de la población

$Z=$ nivel de confianza,

$\mathrm{P}=$ probabilidad de éxito, o proporción esperada

$Q=$ probabilidad de fracaso

$\mathrm{D}=$ precisión (Error máximo admisible en términos de proporción).

Se consideró una seguridad de 95\%, con una precisión de $8 \%$ y una proporción esperada de p = 0.5 (50\%) para maximizar el tamaño de la muestra.

- $Z \alpha 2=1.962$ (ya que la seguridad es del 95\%)

- $p=$ proporción esperada (en este caso $50 \%=0.5$ )

- $q=1-p($ en este caso $1-0.5=0.5)$

- $d$ = precisión (en este caso deseamos un 5\%) quedando como resultado la ecuación (2):

$$
\begin{array}{r}
n=\frac{19771 \times 1,962^{2} \times 0,5 \times 0,5}{0,08^{2} \times(19771-1)+1,962^{2} \times 0,5 \times 0,5} \\
n=149
\end{array}
$$

Como resultado se establece encuestar a 149 empresas familiares. Partiendo de que en Quito el 87,51\% de empresas son de carácter familiar se aumentará un $12.5 \%$ en el total de muestras generando un total de 170 muestras.

\subsection{Instrumentos Aplicados}

Para realizar el levantamiento de información se realiza encuestas postales para obtener, de manera sistemática y ordenada, información sobre las variables que intervienen en este estudio. Esta información hace referencia a lo que las empresas familiares son, hacen, piensan, opinan, sienten, esperan, desean, quieren u odian, aprueban o desaprueban, o los motivos de sus actos, opiniones y actitudes en relación con la cultura del diseño, como estrategia para la innovación y el valor agregado (Díaz de Rada, 2002).

La encuesta es realizada a empresas familiares del área metropolitana de Quito. Sin embargo, el término empresa familiar no ha sido regularizado dentro de los agentes públicos de información dentro del territorio ecuatoriano, por lo que se escoge la lista de empresas de la Superintendencia de Compañía, Valores y Seguros con corte de actualización diciembre de 2019. Para garantizar que la encuesta sea respondida solo por el sector a analizar y evitar los falsos positivos se realiza como primer criterio el valor de caracterización de empresas familiares de acuerdo con el criterio de (Camino \& Bermúdez, Las Empresas Familiares en el Ecuador: Definición y aplicación, 2018) de tal manera que se responda solo en caso de cumplir con el criterio de ser Empresa Familiar.

Los fines perseguidos con el instrumento aplicado es realizar una recolección de información sobre las empresas y la cultura del diseño para posteriormente evaluar su estatus quo para puntuar y ubicar dentro de herramientas de medición internacionales. 
Dentro de las herramientas de medición de la cultura del diseño se utiliza el "Atlas de Diseño" es una herramienta genérica de auditoria del diseño introducida por el Design Council de Reino Unido, puede utilizarse para ayudar a cualquier tipo de organización evaluando su capacidad de diseño. Las preguntas son evaluadas en cinco categorías: planeación, procesos, recursos, personas y Cultura. También se utiliza "La escalera de Diseño" la cual fue desarrollada en 2003 por el Danish Design Centre el modelo pretende graficar los niveles de adopción del diseño por parte de las empresas y al mismo tiempo vincularlo al modelo de gestión de la innovación que utilizan en una escala de medición de forma ascendente (Heinzen, 2017). Además, se utiliza "La Guía Predica" creada por la Sociedad Estatal de Diseño Desarrollo e Innovación, (DDI) de Asturias en España como un modelo basado en diferentes niveles en función de la toma de decisiones respecto a las actividades de diseño para facilitar que su integración (Bernardo, 2011). El objetivo del proyecto PREDICA fue dar a conocer los beneficios del diseño y potenciar su incorporación en las PYMES en cada una de las comunidades autónomas participantes (Asturias, Galicia, Cantabria y Castilla y León) (PRODINTEC, 2011).

Las principales consideraciones de la encuesta se encuentran en el Tabla 1.

Tabla 1

Diseño Especifico del Estudio

FILTRO Pregunta para el filtro de la muestra: Para establecer que solo sea respondida por miembros de Empresas Familiares.

Preguntas filtro de conocimiento del diseño: Basado en que la encuesta es de categorización el nivel más bajo desconoce todos los términos básicos del diseño por lo cual no continuara con las siguientes secciones para evitar sesgos en la investigación.

PRIMERA SECCIÓN: La primera sección busca recabar información de la empresa por CATEGORIZACIÓN DE LA EMPRESA actividad económica, zona, tamaño, nacionalidad, tiempo y nivel de crecimiento.

SEGUNDA SECCIÓN: ANÁLISIS DE LA La segunda sección se establece mediante las herramientas de CULTURA DEL DISEÑO medición de cultura del diseño Escalera de diseño, guía predica y atlas designó a través de preguntas cerradas de elección única (dicotómicas y polínicas) y escala. Su fin es determinar el estado actual en relación con las escalas que ofrecen las herramientas de medición.

TERCERA SECCIÓN: EVALUACIÓN DEL La tercera sección mide mediante preguntas cerradas de elección DISEÑO EN LA GENERACIÓN DE única (dicotómicas y polínicas) la correlación que existe entre la INNOVACIÓN Y VALOR cultura del diseño, la innovación y la generación de valor.

\begin{tabular}{ll}
\hline ANÁLISIS DE BARRERAS DE INGRESO & La sección de barreras de ingreso se establece como una respuesta al \\
DEL DISEÑO & filtro de desconocimiento total del diseño para evaluar posibles causas \\
& de no involucras la cultura del diseño como estrategia de innovación y \\
& generación de valor.
\end{tabular}

Fuente: Elaboración Propia

\section{Resultados}

\subsection{Primera Sección (Categorización de la Empresa)}

Para el tratamiento de las encuestas se consideró una muestra de 170 empresas del área metropolitana de Quito. Luego de aplicar las preguntas de filtro, se depuraron 15 empresas que no cumplieron con la característica de 
ser Empresa Familiar lo que corresponde al 10\% del total de Encuestados. Como resultado se procesaron 153 encuestas.

De las 153 empresas familiares analizadas 87 empresas (56,86\%) son Pequeña Empresa (10-49 trabajadores), 50 empresas (32,86\%) Microempresa (1-9 trabajadores), 14 empresas (9,15\%) son Mediana Empresa (50-99 trabajadores) y 2 empresas 1,31\% Gran Empresa (mayor a 100 trabajadores). A lo que se agrega que de acuerdo con los años de constitución las empresas familiares encuentras despliegan los siguientes resultados: 107 empresas familiares (69,93\%) 1-5 años, 21 empresas familiares (13,73\%) 6-10 años, 11 empresas familiares (7,19\%) menor a 1 año, 8 empresas familiares (5,23\%) mayor a 16 años y 6 empresas familiares (3,92\%) 11-15 años.

\section{Gráfico 1}

Empresas familiares analizadas según la actividad económica

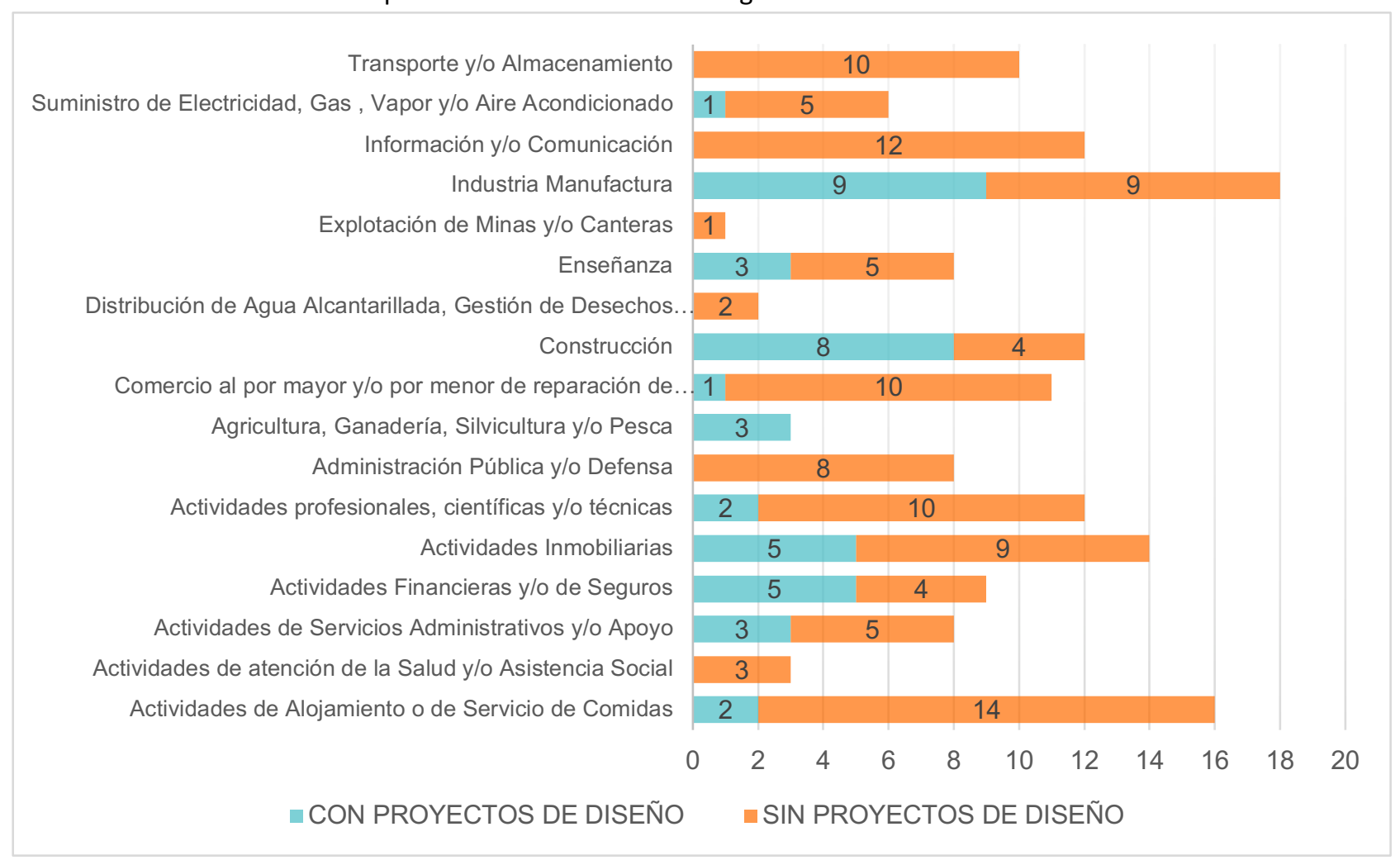

Fuente: Elaboración propia

En el gráfico 1 es posible apreciar que en gran proporción las empresas familiares no cuentan con proyectos de diseño. Solo un pequeño grupo de empresas familiares cuenta con una gestión enfocada a los proyectos de diseño. Se puede observar que dentro de las actividades comerciales analizadas la industria manufacturera, la construcción, las actividades inmobiliarias y las actividades financieras y/o de seguros presentan mayor número de empresas con proyectos de diseño. Sin embargo, el número total de encuestas por actividad es dispar y con una muestra no adecuada para marcar una tendencia.

Se evaluaron los pilares competitivos de las empresas, tomando en cuenta su pertinencia en la cultura del diseño, la tabla 1 muestra los resultados obtenidos en este respecto. 
Tabla 2

Pilares Competitivos de las Empresas Familiares

\begin{tabular}{lcc}
\hline PILARES COMPETITIVOS & Frecuencia & Porcentaje \\
\hline Liderazgo en costos & 91 & $59,48 \%$ \\
\hline Diferenciación en el servicio al cliente & 37 & $24,18 \%$ \\
\hline Capacidad productiva & 30 & $19,61 \%$ \\
\hline Diferenciación en tecnología & 29 & $18,95 \%$ \\
\hline Diferenciación en diseño de producto & 21 & $13,73 \%$ \\
\hline Diferenciación en imagen de marca & 17 & $11,11 \%$ \\
\hline Correcta delimitación del público objetivo & 17 & $11,11 \%$ \\
\hline No considero tener pilares competitivos & 5 & $3,27 \%$ \\
\hline
\end{tabular}

Fuente: Elaboración Propia

En la evaluación a las empresas familiares fue posible observar que establecen como sus principales pilares competitivos el liderazgo en costo, la diferenciación en el servicio al cliente y la capacidad productiva. La diferenciación por diseño de productos e imagen de marca ocupan los lugares más bajos con la correcta delimitación del público objetivo. Los resultados presumen que las empresas familiares analizadas priorizan la atención, el volumen y el precio, lo que representaría poca priorización en el desarrollo del producto. Esto datos podrían presumir una baja inserción de cultura de diseño como estrategia ya que no se considera la innovación, novedad o valor agregado como una fuente de competitividad de las empresas.

También fue posible observar que las empresas familiares consideran su crecimiento estable, a lo que se suma un $35.29 \%$ que considera estar en crecimiento, un $13,73 \%$ que considera que el crecimiento es inestable y un porcentaje mínimo del $0,65 \%$ considera que es decreciente. Los resultados presumen que las empresas se encuentran en posiciones estables de crecimiento lo que indicaría que se mantienen dentro de la realización habitual de sus productos o servicios. Esta respuesta a un nivel de crecimiento estable podría indicar que no existe un nivel adecuado de estrategias gerenciales para asegurar el crecimiento efectivo y eficaz.

Otro factor importante es que las empresas familiares consideran desenvolverse en un nivel de competitividad de mercado estable con $32,03 \%$ y competitivo $29,41 \%$, estos dos valores marcan la mayor cantidad de respuestas lo que ubica el mercado general de las empresas familiares en un mercado relativamente competitivo. Estos datos podrían establecer que el nivel de competencia del mercado actual no está retando a cambios periódicos dentro de las Empresas, y presumiría una relajación dentro del mercado.

\subsection{Segunda Sección (Análisis de la Cultura del Diseño)}

Para evaluar mediante herramientas internacionales el uso de la cultura del diseño, se aplicó un segundo filtro que determine que empresas familiares utilizar el diseño dentro de sus procesos. De las 153 Empresas Familiares analizadas 111 declararon no utilizar el diseño dentro de sus procesos y 42 si lo involucran. Por consecuente, el análisis de la cultura del diseño se aplicó a las 42 Empresas Familiares y se presentan los siguientes resultados:

Los niveles de cultura del diseño bajo la escalera de diseño: de las 42 empresas familiares del área metropolitana de Quito que involucran diseño se distribuyen en $14,38 \%$ en Sin Diseño, un $13,07 \%$ utiliza el Diseño como Formulario, un $0,65 \%$ utiliza el Diseño como Proceso y un $0,65 \%$ utiliza el Diseño como Estrategia. 


\section{Gráfico 2}

Porcentaje de Empresas Analizadas en

los niveles de la Escalera de Diseño

\section{ESCALERA \\ DE DISEÑO}

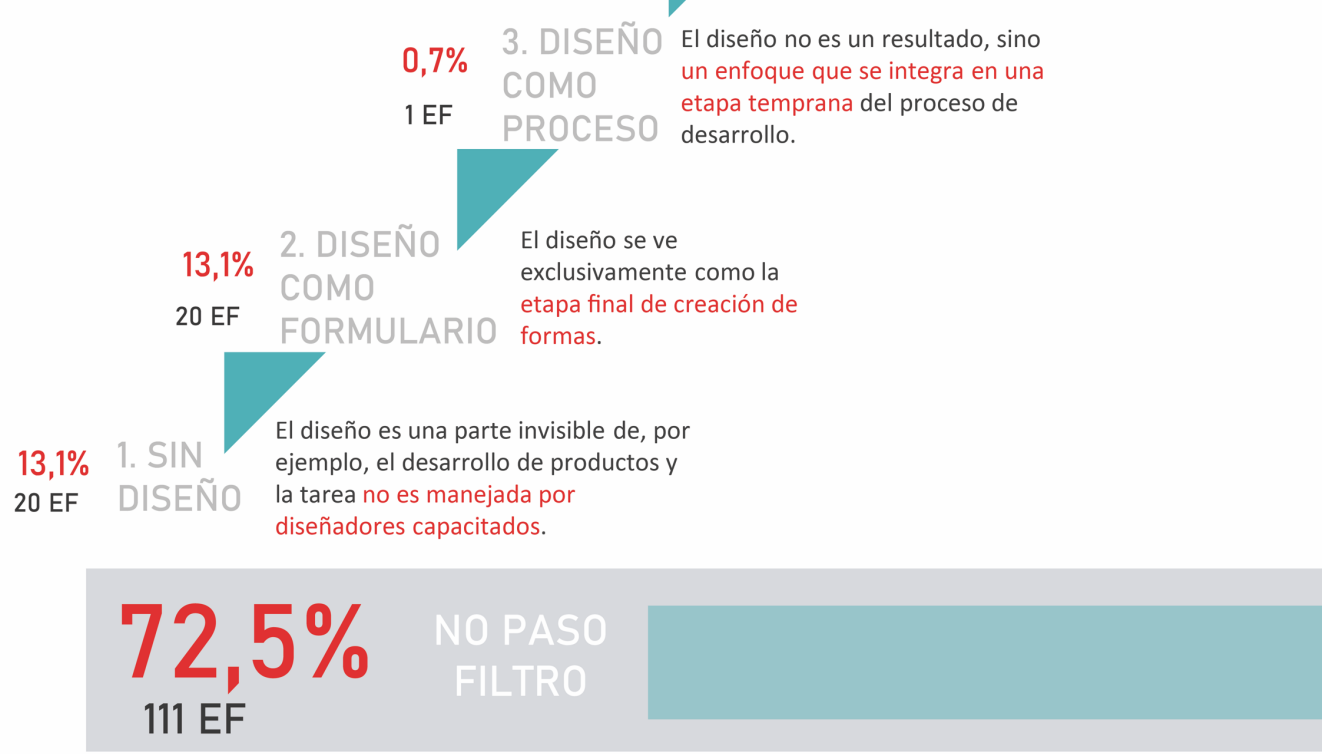

Fuente: Elaboración Propia basado en (Danish Design Centre, 2015)

Los niveles de cultura del diseño bajo la Guía Predica de las 42 empresas familiares que involucran diseño del área metropolitana de Quito ubican al 15,69\% en el Nivel 1, al 7,19\% en el Nivel 2, al 1,31\% en el Nivel 3 y el $3,27 \%$ en el Nivel 4. 
Gráfico 3

Porcentaje de Empresas Analizadas

en los niveles de la Guía Predica

\section{GUÍA \\ PREDICA}

El diseño se integra en los valores de la empresa. Es el que rige y regula sus actividades
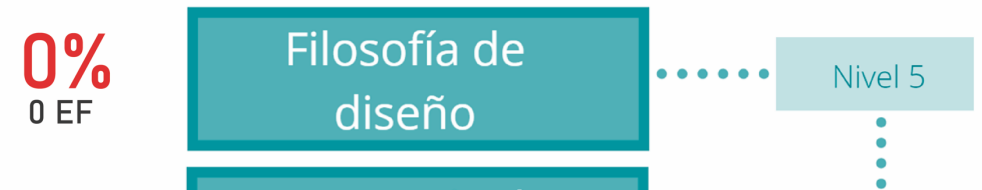

Estrategia competitiva
basada en diseño de producto, comunicación
de producto e imagen
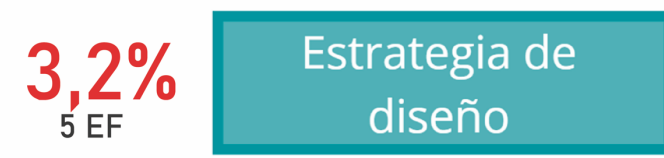

Se definen los medios con los que poner en práctica estrategias basadas en el diseño.

Se generan planes estructurados con fases y objetivos.

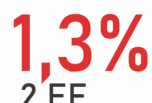

2 EF

7, $1 \%$

Se realizan ocasionalmente actividades de diseño en distintos proyectos
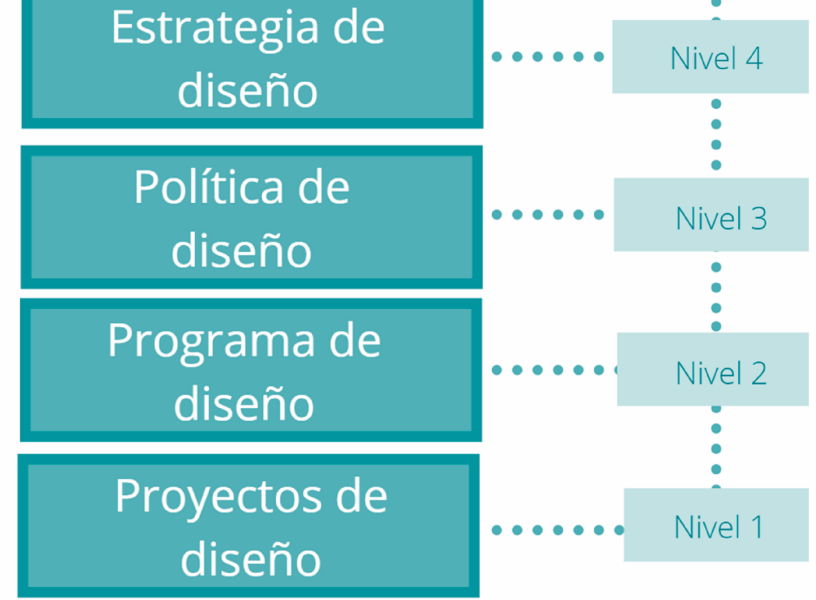

\section{$72,5 \%$ $111 \mathrm{EF}$}

Fuente: Elaboración Propia basado en (Bernardo, 2011)

Los niveles de cultura del diseño bajo el Atlas Design establecen que las empresas familiares que involucran diseño del área metropolitana de Quito logran en un 10,46\% cumplir con el aspecto de Planeación del Diseño y un $16,34 \%$ con los Recursos, ninguna empresa logro cubrir los aspectos de Procesos, Personas y Cultura para el Diseño. 
Gráfico 3

Porcentaje de Empresas Analizadas

por criterios del Atlas Design

\section{ATLAS \\ DESIGN}

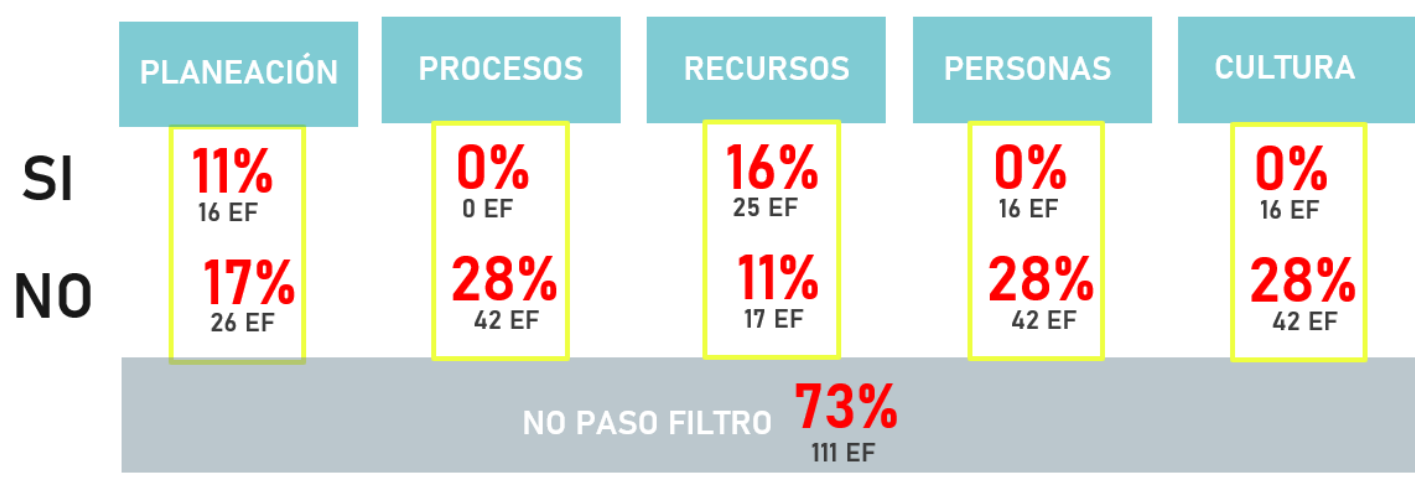

Fuente: Elaboración Propia

basado en (Holland \& Lam, 2014)

\subsection{Tercera Sección (Evaluación del diseño en la generación de innovación y valor)}

En esta sección se obtienen los resultados de las 42 empresas familiares que involucran procesos de diseño dentro de sus organizaciones. De estas las 33 empresas familiares $(78,57 \%)$ considera que sus procesos de innovación se deben a la inversión en diseño y 9 empresas familiares $(21,43 \%)$ no considera que tenga incidencia.

Con un $74 \%$ las empresas familiares consideran adecuado el retorno de la inversión de los proyectos de innovación por diseño y un $26 \%$ no considera adecuado el retorno. Además un $90 \%$ de estas empresas consideran que el proceso de diseño aporta al producto/servicio un mayor índice de funcionalidad frente a un $10 \%$ que no lo considera.

\subsection{Cuarta Sección (Análisis de barreras de ingreso del diseño)}

Dentro de esta sección se evalúa a las 111 empresas familiares (73\%) que no involucran procesos de diseño. Estas empresas con un $71,17 \%$ establecen que desconocen cómo podría involucrarse el diseño dentro de su empresa y un $28,83 \%$ establece saber cómo involucrarlo. Otro aspecto relevante es que se atribuye la no contratación del diseño a los siguientes factores: En un $41,83 \%$ por los costos que implica, $21,57 \%$ no conoce las funciones del diseño, 20,26\% por mala experiencia con contrataciones anteriores, 15,03\% considera que no es necesario y $4,58 \%$ resuelve las necesidades de diseño con el personal existente.

\section{Conclusiones}

Dentro de las 153 Empresas Familiares estudiadas se encontró que 111 empresas no involucran procesos de diseño (72,55\%) y 42 si lo involucran (27,45\%). Las 153 empresas familiares relacionan como pilares competitivos a el liderazgo en costos $(59,18 \%)$, atención al cliente $(24,18 \%)$ y volumen de producción $(19,61 \%)$. Mientras que las características competitivas asociadas al diseño se encuentran con niveles por debajo del $18 \%$, lo que podría representar que las empresas priorizan la inversión en características de manufactura y servicio versus la ideación y diseño. 
Dentro del análisis del nivel de cultura de Diseño de las 42 Empresas Familiares que utilizan el diseño dentro de sus procesos se obtiene que se ubican principalmente dentro de los dos primeros niveles en la Guía Predica (89\%) y Escalera del Diseño (86\%). Lo que supondría que el nivel de cultura de diseño dentro de estas empresas no

Dentro del análisis de las 42 Empresas Familiares bajo el Atlas Design se desprende que se cumplen dos de los cinco aspectos. Planeación con un $11 \%$ y Recursos $16 \%$, los aspectos de Personas, Cultura y Procesos no presentan valores positivos. Lo que sugiere que el diseño se presenta como una etapa final con características netamente estéticas y que no se realiza un proceso de ideación y análisis adecuado para el lanzamiento de nuevos productos o servicios.

Las Empresas Familiares que no involucran procesos de diseño (111) presentan un gran desconocimiento del beneficio de la cultura del diseño y de la gestión empresarial a través de este. Dichas empresas atribuyen a los principales motivos de no adquirir servicios de diseño a los costos que implica $(41,83 \%)$ y refieren desconocer como el diseño puede involucrarse en su empresa (21,57\%). Es así como se estimaría que los rubros asignados al diseño son visualizados por los tomadores de decisiones de las empresas como una característica de poco valor. Además, estos valores se pueden reflejar en productos que no analizan las necesidades del usuario final, generando un valor agregado mínimo y poca o nula innovación.

Las Empresas Familiares que involucran procesos de diseño (42), refieren que el retorno de la inversión de los proyectos de diseños es adecuado en un $74 \%$ y en un $90 \%$ consideran que el proceso de diseño aporta al producto/servicio un mayor índice de funcionalidad. Aunque la muestra es reducida podría analizarse que el entendimiento y uso de las bondades y campos de acción del diseño pueden incidir directamente en el crecimiento de la utilidad.

De las 153 Empresas Familiares analizadas dentro de este estudio, en mayor proporción consideran encontrarse en un mercado con niveles de competitividad bajos. Estos resultados podrían significar las causas principales por las cuales no se encuentra un nivel de la Cultura del Diseño como estrategia de innovación y de Gestión Empresarial en niveles adecuados. Se podría analizar la posibilidad de una correlación entre la competitividad y el uso del diseño.

\section{Bibliografía}

Balmaseda, E., \& Zamanillo, I. (2008). EVOLUCIÓN DE LAS PROPUESTAS SOBRE EL PROCESO DE INNOVACIÓN: ¿QUÉ SE PUEDE CONCLUIR DE SU ESTUDIO? Investigaciones Europeas de Dirección y Economía de la Empresa, 127-138. Obtenido de ScienceDirect.

Barcelona Escola Superior de Disseny. (25 de Mayo de 2016). La importancia del diseño para acelerar la innovación. Obtenido de Hipertextual: https://hipertextual.com/presentado-por/ied-barcelona/ideascreativas

Bernardo, C. (Abril de 2011). La Gestión del Diseño según la Guía metodológica PREDICA. Obtenido de Dialnet Universidad de La Rioja: https://dialnet.unirioja.es/servlet/articulo?codigo $=4550738$

Camino, S., \& Bermudez, N. (2018). Las Empresas Familiares en el Ecuador: Definición y aplicación. X-Pedientes Económicos, $\mathrm{Vol} 2$, 46-72.

Cañate, N., \& Basco, I. (12 de Junio de 2018). ¿Es la innovación un tema relevante para el desarrollo? Esto es lo que piensan los latinoamericanos. Obtenido de Banco Interamericano de Desarrollo: https://blogs.iadb.org/innovacion/es/innovacion-en-la-agenda-para-el-desarrollo/ 
Chaves, N. (24 de Septiembre de 2016). Diseño e innovación. Obtenido de Archivo de Norberto Chaves: https://www.norbertochaves.com/articulos/texto/diseno_e_innovacion

Danish Design Centre. (6 de Mayo de 2015). THE DESIGN LADDER: Four steps of design use. Obtenido de Danish Design Centre: https://danskdesigncenter.dk/en/design-ladder-four-steps-design-use

DESARROLLO MIC. (27 de Abril de 2017). ¿Por qué es importante la innovación en las organizaciones? Obtenido de Infórmese. We make analytucs Happen: https://www.informese.co/innovacion-en-organizaciones/

Díaz de Rada, V. (2002). Tipos de encuestas y diseños de investigación. Obtenido de Catálogo de Publicaciones de la Universidad Pública de Navarra: http://www.unavarra.es/personal/vidaldiaz/pdf/tipos_encuestas.PDF

El Telégrafo. (28 de Noviembre de 2017). En el país, el 90,5\% de empresas son familiares. Obtenido de El Telégrafo: https://www.eltelegrafo.com.ec/noticias/economia/4/en-el-pais-el-90-5-de-empresas-sonfamiliares

Fernández, S. (04 de Abril de 2017). Si las piedras hablaran. Metodología cualitativa de Investigación en. Obtenido de Revista la Razón Historica: https://www.revistalarazonhistorica.com/app/download/11357684798/Sergio+Fern\%C3\%A1ndez+Riquel me.+Metodolog\%C3\%ADa+cualitativa.+La+Raz\%C3\%B3n+hist\%C3\%B3rica+.pdf?t=1529403668\&mobile=1

Fontalvo, A., Luckert, A., Martínez, S., \& Olivella, J. (14 de Agosto de 2013). Latin American and Caribbean Conference for Engineering and Technology (LACCEI'2013). Obtenido de Papel de la innovación en el sector empresarial: http://www.laccei.org/LACCEI2013-Cancun/StudentPapers/SP016.pdf

Gay, A., \& Samar, L. (2007). El diseño industrial. Editorial TEC.

Gómez, \& Yaffa. (Agosto de 2010). La Cultura del Diseño, estrategia para la generación de valor e innovación en la PyMe del Área Metropolitana del Centro Occidente, Colombia. Obtenido de Plataforma Diguital Cuadernos del Centro de Estudios en Diseño y Comunicación: https://fido.palermo.edu/servicios_dyc/publicacionesdc/cuadernos/detalle_articulo.php?id_libro=165\&id _articulo=6163

González, J. (18 de Agosto de 2013). Innovar: un imperativo para competir en la economía mundial. Obtenido de Scielo: http://www.scielo.org.co/pdf/ring/n38/n38a09.pdf

Heinzen, A. (14 de Noviembre de 2017). LA ESCALERA DEL DISEÑO, ESTRATEGIAS DE INNOVACIÓN. Obtenido de Kairos \& Cronos SRL: http://www.kyc.com.uy/2017/11/14/la-escalera-del-diseno/

Holland, R., \& Lam, B. (2014). Managing Strategic Design. Inglaterra: PALGRAVE.

Instituto Nacional de Estadística y Censos. (2016). Encuesta Nacional de Actividades de Innovación (Al): 20122014. Obtenido de Ecuador en Cifras: https://www.ecuadorencifras.gob.ec/documentos/webinec/Estadisticas_Economicas/Ciencia_Tecnologia-ACTI/20122014/Innovacion/Metodologla\%20INN\%202015.pdf

InVision. (27 de Octubre de 2018). Design culture. Obtenido de InVision : https://www.invisionapp.com/designdefined/design-culture/

Iváñez, J. M. (2000). La gestion del diseño en la empresa. En J. M. Iváñez, La gestion del diseño en la empresa (págs. 4-7). Madrid: McGRAW-HILL/INTERAMERICANA DE ESPAÑA, S. A. U. 
Jewell, C. (07 de Junio de 2016). Sacar a la luz el potencial de diseño oculto en los países en desarrollo. Obtenido de OMPI Revista: https://www.wipo.int/wipo_magazine/es/2016/03/article_0002.html

Martinez, C. (05 de Mayo de 2020). Investigación descriptiva: definición, tipos y características. Obtenido de Lifeder: https://www.lifeder.com/investigacion-descriptiva/

Organización Mundial de la Propiedad Intelectual. (16 de Noviembre de 2015). Aprovechar el potencial de diseño de un país. Obtenido de OMPI: https://www.wipo.int/edocs/mdocs/stlt/es/wipo_wk_Idcs_ge_15/wipo_wk_ldcs_ge_15_brochure.pdf

Organización Mundial de la Propiedad Intelectual. (24 de Julio de 2019). Índice Mundial de Innovación 2019. Obtenido de OMPI: https://www.wipo.int/export/sites/www/pressroom/es/documents/pr_2019_834_annex1.pdf

Ortt, J., \& Smits, R. (2006). Innovation management: different approaches to cope with the same trends. International Journal of Technology Management Vol. 34, 296-318.

PRODINTEC. (2011). Guía metodológica PREDICA. Obtenido de PRODINTEC: http://www.prodintec.es/es/capacidades-y-experiencia/publicaciones/293-guia-metodologica-predica

Rodríguez, N. (1999). La Innovación: Clave del Éxito Empresarial. La gestión de la diversidad: XIII Congreso Nacional, IX Congreso Hispano-Francés (págs. 251-256). La Rioja: Universidad de La Rioja. Obtenido de Universidad de La Rioja.

Romero, V. (10 de Agosto de 2017). ¿Por qué es importante generar un valor agregado en los negocios? Obtenido de Ruiz Healy Times: https://www.ruizhealytimes.com/economia-y-negocios/por-que-esimportante-generar-un-valor-agregado-en-los-negocios

Ruíz, M. (26 de Junio de 2020). La empresa familiar y su necesidad de ser competitiva. Obtenido de Murcia Economía: https://murciaeconomia.com/art/64402/la-empresa-familiar-y-su-necesidad-de-sercompetitiva

Segundo, C. (25 de Agosto de 2019). El reto de la productividad empresarial en Ecuador. Obtenido de Primicias: https://www.primicias.ec/noticias/firmas/el-reto-de-la-productividad-empresarial-en-ecuador/

Simón, G. (19 de Diciembre de 2011). Por qué el diseño es indispensable para las empresas. Obtenido de FOROALFA.COM: https://foroalfa.org/articulos/por-que-el-diseno-es-indispensable-para-las-empresas

Soliz, N., Mena, V., \& Núñez, T. (2017). Cultura y Gestión del Conocimiento en organizaciones del Ecuador. Revista Publicando, 70-83. Obtenido de Revista Publicando.

Vélez, D. (06 de Junio de 2008). Innovación y tradición en las empresas familiares. Obtenido de Dinero: https://www.dinero.com/columnistas/edicion-impresa/articulo/innovacion-tradicion-empresasfamiliares/64096

Esta obra está bajo una Licencia Creative Commons Attribución-NoCommercial 4.0 International

(c) $\mathbf{B Y}$-NC 